\title{
Antibiotic usage in surgical prophylaxis: a prospective surveillance of surgical wards at a tertiary hospital in Malaysia
}

\author{
Ai Ling Oh ${ }^{1}$, Leh Min Goh ${ }^{1}$, Nik Abdullah Nik Azim², Chee Sian Tee², Chee Wei Shehab Phung ${ }^{2}$ \\ ${ }^{1}$ Department of Pharmacy, Sarawak General Hospital, Sarawak, Malaysia \\ ${ }^{2}$ Department of Surgery, Sarawak General Hospital, Sarawak, Malaysia
}

\begin{abstract}
Introduction: The widespread and inappropriate use of broad-spectrum antibiotics in surgical prophylaxis has led to reduced treatment efficacy, increased healthcare costs, and antibiotic resistance. This study aimed to explore the adherence of antibiotic usage in surgical prophylaxis to the national antibiotic guideline and the incidences of surgical site infection (SSI).

Methodology: A three-month prospective observational study has been conducted in the surgical wards of Sarawak General Hospital (SGH) using a standardized surveillance form. Each patient was reviewed for up to 30 days post-operatively to determine the occurrence of SSI.

Results: A total of 87 patients were included within the study period. The majority of the cases were clean-contaminated wounds (60.9\%). Most were hepatobiliary cases (37.9\%), followed by colorectal cases $(19.5 \%)$. The most preferred antibiotic used was cefoperazone (63.2\%). The choices of antibiotics in $78.2 \%$ of the cases were consistent with the guideline. Around $80 \%$ of prophylactic antibiotics were given within one hour before operation and $27.6 \%$ were omitted from intraoperative re-dosing. Prophylactic antibiotics were discontinued within 24 hours post-operatively in $77 \%$ of the cases. Of those continued for $>24$ hours, the majority $(60 \%)$ were administered for unknown reasons. SSI was documented in $13.8 \%$ of the total cases studied. However, there was no significant association between choices of antibiotics and timing of surgical prophylaxis with SSI ( $\mathrm{p}=0.299$ and $\mathrm{p}=0.258$ respectively).

Conclusion: Overall guideline adherence rate was more than $70 \%$. Areas of non-concordance to the guideline require further investigation..
\end{abstract}

Key words: antibiotic; surgical prophylaxis; surveillance, Malaysia

J Infect Dev Ctries 2014; 8(2):193-201. doi:10.3855/jidc.3076

(Received 13 October 2012 - Accepted 27 March 2013)

Copyright $\odot 2014$ Oh et al. This is an open-access article distributed under the Creative Commons Attribution License, which permits unrestricted use, distribution, and reproduction in any medium, provided the original work is properly cited.

\section{Introduction}

Antibiotic prophylaxis, a very brief course of antibiotics initiated just before the start of surgical procedures [1] in clean and clean-contaminated surgery, is recommended to reduce postoperative infection. The practice of giving antibiotic prophylaxis has resulted in the reduction of surgical site infections, thus reducing cost, morbidity, and mortality [2,3]. However, the widespread and prolonged use of broadspectrum antibiotics has contributed to increased healthcare costs and risks of antibiotic resistance, while inappropriate timing of antibiotics administration has shown to reduce antibiotic efficacy [4,5].

The variable practices in surgical prophylaxis have been reported widely; the variation in practice could be attributed to the considerable variation in the published guidelines, especially regarding the timing and duration of antibiotic prophylaxis, the lack of agreement among the surgeons with the guideline, and the lack of awareness and distribution of the guideline
[6,7]. According to a study conducted in Dutch hospitals, overall guideline adherence in surgical prophylaxis was difficult to achieve and was found to be only $28 \%$ [7]. Thus, in order to reduce postoperative infection attributable to antibiotic prophylaxis, inappropriate use of antibiotics has to be considered in order to achieve an overall reduction in healthcare cost, morbidity, and mortality.

As part of quality improvement in antibiotic usage, we explored the adherence status to the national antibiotic guideline in surgical prophylaxis. In our setting, Sarawak General Hospital, a tertiary referral center in the Sarawak state of Malaysia, there was no local hospital guideline pertaining to this area; thus, adherence was assessed based on the aspects delineated in the National Antibiotic Guideline 2008 [8]. 


\section{Methodology}

\section{Study design and ethical consideration}

This prospective observational study was conducted in the general surgical wards of Sarawak General Hospital (SGH) over a three-month period between 1 July and 30 September 2011. The research has been approved by the Medical Research and Ethics Committee (MREC), Ministry of Health Malaysia with the registration ID NMRR-11-414-9547.

A standardized surveillance form was used to facilitate data collection; all patients were selected using a universal sampling method. Patients who were older than 18 years of age, did not have documented infections before surgery, had wounds classified as clean and clean-contaminated, and underwent elective surgical procedures were eligible for inclusion in this study.

\section{Data collection}

All relevant data were retrieved from the patients' medication charts and medical records. Details recorded included patients' demographic data, antibiotic allergy, type of surgery, wound class (clean and clean-contaminated), duration of operation, and parameter of antibiotic prophylaxis (antibiotic choice, route, dose, timing and duration of prophylaxis). If antibiotics were to be continued for more than 24 hours after surgery, the reasons for the prolonged usage were explored. Each patient was reviewed from the time of admission until their discharge from the hospital, and subsequent follow-up at the outpatient clinic was scheduled up to 30 days postoperatively for surgical site inspection. For those patients who defaulted or missed the follow-up, the determination of the occurrence of surgical site infection (SSI) was done via telephone interview. This study was a collaborative work between the Pharmacy and the Surgical Department of Sarawak General Hospital; clinical pharmacists and medical officers were involved in the data collection, and surgeons were involved in the wound classification and surgical site inspection. The wound classification and SSI criteria were based on the Centers for Disease Control (CDC) standards [9]. SSI is considered when there is at least one of the following: redness, edema, tenderness, gaping, abscess or purulent discharge, occurrence of fever $\left(>38^{\circ} \mathrm{C}\right)$, or positive culture of fluid or tissue from the surgical site within 30 days of the operation.

\section{Guideline recommendations}

According to the National Antibiotic Guideline 2008, antibiotic prophylaxis, when given intravenously, should be given as soon as the patient is stabilized after induction. A single dose is usually sufficient. In situations where there is a delay in start of surgery and in prolonged operations when the time is more than half of the usual dosing interval of the antibiotic, a second dose may be required. Postoperatively administering more than one or two doses is generally not advised. Additionally, continuing prophylactic antibiotics until surgical drains have been removed is not recommended [8]. As there was no specific period recommended for the timing and duration of surgical prophylaxis in the guideline, antibiotic prophylaxis was considered to have been given appropriately based on the performance measures in the Policies and Procedures on Infection Control, Ministry of Health, developed in 2010 [10]. There are three performance measures recommended for antibiotic prophylaxis surveillance: (1) the proportion of patients who received appropriate prophylactic antibiotic is consistent with the national antibiotic guideline (Table 1), (2) the proportion of patients who received antibiotic prophylaxis within one hour preceding surgical incision, and (3) the proportion of patients who received antibiotic prophylaxis for $<24$ hours. Adherence to these measures is considered appropriate practice. If more than one drug was prescribed for a single procedure, all parameters were evaluated separately for each drug. Divergences of one of the drugs in the prescription led to a final assessment as discordant with the national guideline.

\section{Statistical analysis}

All data collected were analyzed using the Statistical Package for the Social Sciences (SPSS Inc., Chicago, USA) version 17.0. Descriptive statistic was used to summarize the demographic characteristics, surgical information, and antibiotic usage data. The association between usage of antibiotic in surgical prophylaxis and SSI was analyzed using Fisher's exact test. The level of statistical significance was set at $\mathrm{p}<$ 0.05 . 
Table 1. Recommended antibiotics according to the type of surgery (adapted from National Antibiotic Guideline 2008, Ministry of Health Malaysia)

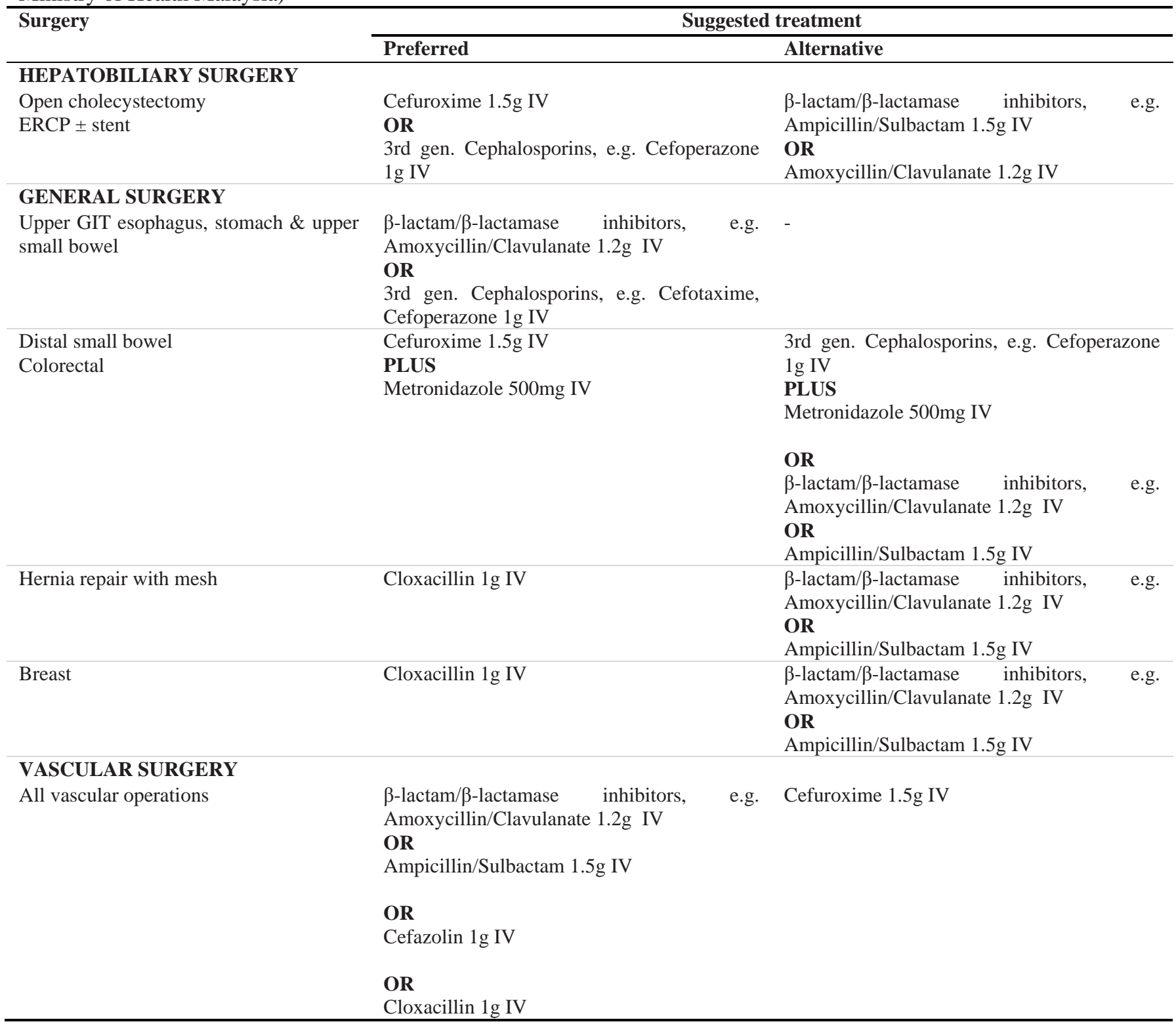




\section{Results}

A total of 87 patients who underwent elective surgical procedures fulfilled the criteria to be included in the study. The mean age of the cohort was 54.2 (SD $=14.7$ ), and approximately $60 \%$ of the patients were females. There were $53(60.9 \%)$ clean-contaminated operations and $34(39.1 \%)$ clean operations that involved the use of antibiotics as surgical prophylaxis. Of all surgical procedures performed, $37.9 \%$ were hepatobiliary, $19.5 \%$ were colorectal, $11.5 \%$ were vascular, $9.2 \%$ were hernia, and $8.1 \%$ each were upper gastrointestinal (esophageal and gastroduodenal) and breast surgery (Table 2).

There were four main types of antibiotics used in the study, with cefoperazone used most (63.2\%), followed by metronidazole $(40.2 \%)$, augmentin $(32.2 \%)$, and cefuroxime (4.6\%). The combination of cefoperazone and metronidazole was seen most frequently in the present cohort, in 33 cases (37.9\%). Cefoperazone with or without metronidazole was mainly used for the hepatobiliary, colorectal, upper gastrointestinal (esophageal and gastroduodenal) cases, whereas augmentin, which was used in approximately one-third of the cases, was mainly used in vascular, hernia, and breast surgery. There were 19 cases $(21.8 \%)$ found to be discordant with the recommended choice of antibiotics stated in the national guideline, mostly due to the addition of metronidazole to the antibiotics regimen in hepatobiliary $(13 / 19 ; 68.4 \%)$ and upper gastrointestinal (esophageal and gastroduodenal) operations $(4 / 19 ; 21.1 \%)$. All the antibiotics were administered via intravenous route and the doses used were concordant with the national antibiotic guideline (Tables 3 and 4).

In terms of the choice of antibiotics and incidences of SSI (Table 5), the use of cefoperazone with metronidazole in hepatobiliary surgery (no recommendation for the use of metronidazole in the guideline) yielded higher incidences of SSI (3/12; $25 \%)$ as compared to cefoperazone alone (3/18; $16.7 \%)$.

Table 2. Demographic characteristics of patients and surgical information

\begin{tabular}{|c|c|c|}
\hline Characteristics & Number $(\mathbf{N}=87)$ & Percentage (\%) \\
\hline $\begin{array}{l}\text { Age } \\
\text { Mean (SD) }\end{array}$ & $54.2(14.7)$ & \\
\hline $\begin{array}{l}\text { Gender } \\
\text { Male } \\
\text { Female }\end{array}$ & $\begin{array}{l}35 \\
52\end{array}$ & $\begin{array}{l}40.2 \\
59.8\end{array}$ \\
\hline $\begin{array}{l}\text { Race } \\
\text { Malay } \\
\text { Chinese } \\
\text { Iban }\end{array}$ & $\begin{array}{l}19 \\
33 \\
35\end{array}$ & $\begin{array}{l}21.8 \\
37.9 \\
40.2\end{array}$ \\
\hline $\begin{array}{l}\text { Wound classification } \\
\text { Clean } \\
\text { Clean-contaminated }\end{array}$ & $\begin{array}{l}34 \\
53\end{array}$ & $\begin{array}{l}39.1 \\
60.9\end{array}$ \\
\hline $\begin{array}{l}\text { Type of surgery } \\
\text { Hepatobiliary } \\
\text { Colorectal } \\
\text { Vascular } \\
\text { Hernia } \\
\text { Esophageal, gastroduodenal } \\
\text { Breast } \\
\text { Others }\end{array}$ & $\begin{array}{l}33 \\
17 \\
10 \\
8 \\
7 \\
7 \\
5\end{array}$ & $\begin{array}{l}37.9 \\
19.5 \\
11.5 \\
9.2 \\
8.1 \\
8.1 \\
5.7\end{array}$ \\
\hline $\begin{array}{l}\text { Duration of surgery (in hours) } \\
<1 \\
1 \text { to } 2 \\
>2 \text { to } 4 \\
>4 \text { to } 6 \\
>6\end{array}$ & $\begin{array}{c}8 \\
32 \\
22 \\
11 \\
14\end{array}$ & $\begin{array}{c}9.2 \\
36.8 \\
25.3 \\
12.6 \\
16.1\end{array}$ \\
\hline $\begin{array}{l}\text { Surgical site infection } \\
\text { Yes } \\
\text { No }\end{array}$ & $\begin{array}{l}12 \\
75\end{array}$ & $\begin{array}{l}13.8 \\
86.2\end{array}$ \\
\hline
\end{tabular}


Table 3. Parameters of antibiotic usage

\begin{tabular}{lcc}
\hline Parameters & Number $(\mathbf{N}=\mathbf{8 7})$ & Percentage $(\%)$ \\
\hline Choices of Antibiotics & & 22 \\
Cefoperazone 1g IV & 33 & 25.3 \\
Cefoperazone 1g + Metronidazole 500mg IV & 28 & 37.9 \\
Amoxycillin/Clavulanate 1.2g IV & 2 & 32.2 \\
Cefuroxime 1.5g IV & 2 & 2.3 \\
Cefuroxime 1.5g + Metronidazole 500mg IV & & 2.3 \\
\hline Timing (in hours) & 4 \\
-1 to $<-0.5$ & 66 & 4.6 \\
-0.5 to $<0$ & 13 & 75.9 \\
0 (incision) & 4 & 14.9 \\
$>0$ to 0.5 & & 4.6 \\
Duration of antibiotic prophylaxis (in hours) & 67 & 77.0 \\
Prophylaxis $(\leq 24$ hrs) & 20 & 23.0 \\
Therapeutic $(>24$ hrs) & & \\
\hline
\end{tabular}

Table 4. Choices of antibiotics according to the type of surgery $(\mathrm{N}=87)$

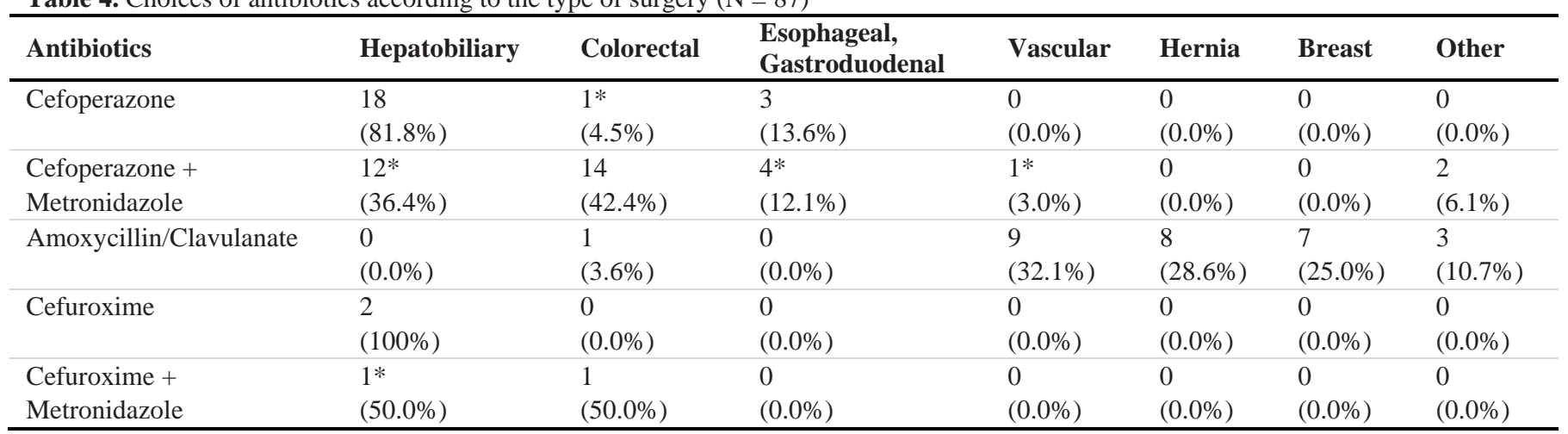

*Discordant of the selection of antibiotics according to the National Antibiotic Guideline 2008, Ministry of Health Malaysia

Table 5. Incidences of SSI pertaining to the choice of antibiotics and type of surgery $(\mathrm{N}=87)$

\begin{tabular}{|c|c|c|c|c|c|c|c|c|c|c|c|c|c|c|}
\hline \multirow{3}{*}{ Antibiotics } & \multicolumn{14}{|c|}{ Number of cases with surgical site infection (SSI) } \\
\hline & \multicolumn{2}{|c|}{ Hepatobiliary } & \multicolumn{2}{|c|}{ Colorectal } & \multicolumn{2}{|c|}{$\begin{array}{c}\text { Esophageal, } \\
\text { Gastroduodenal }\end{array}$} & \multicolumn{2}{|c|}{ Vascular } & \multicolumn{2}{|c|}{ Hernia } & \multicolumn{2}{|c|}{ Breast } & \multicolumn{2}{|c|}{ Other } \\
\hline & Yes & No & Yes & No & Yes & No & Yes & No & Yes & No & Yes & No & Yes & No \\
\hline $\begin{array}{l}\text { Cefoperazone + } \\
\text { Metronidazole }\end{array}$ & 3 & 9 & 2 & 12 & 1 & 3 & 0 & 1 & 0 & 0 & 0 & 0 & 0 & 2 \\
\hline Amoxycillin/Clavulanate & 0 & 0 & 0 & 1 & 0 & 0 & 0 & 9 & 0 & 8 & 0 & 7 & 0 & 3 \\
\hline $\begin{array}{l}\text { Cefuroxime }+ \\
\text { Metronidazole }\end{array}$ & 0 & 1 & 0 & 1 & 0 & 0 & 0 & 0 & 0 & 0 & 0 & 0 & 0 & 0 \\
\hline
\end{tabular}

Table 6. Surgical site infection (SSI)

\begin{tabular}{lcc}
\hline SSI presentation & Number of cases $(\mathbf{N}=\mathbf{1 2})$ & Percentage $(\%)$ \\
\hline Redness & 10 & 83.3 \\
Tenderness & 9 & 75.0 \\
Edematous & 1 & 8.3 \\
Gaping & 1 & 8.3 \\
Abscess/purulent discharge & 2 & 16.7 \\
Fever $\left(>38^{\circ} \mathrm{C}\right)$ & 2 & 16.7 \\
Positive culture (fluid/tissue) & 2 & 16.7 \\
\hline
\end{tabular}


In esophageal and gastroduodenal surgery, the combination of cefoperazone with metronidazole produced fewer SSI incidences $(1 / 4 ; 25 \%)$ as compared to cefoperazone alone $(2 / 3 ; 66.7 \%)$, even though the use of metronidazole deviated from the guideline. However, there was no significant association between choices of antibiotics with SSI $\left(\chi^{2}\right.$ $=1.077, \mathrm{p}=0.299$ ).

As for the timing of antibiotic administration, around three quarters $(75.9 \%)$ of the antibiotics were administered within 30 minutes of the start of the operation. The remaining $4.6 \%$ received prophylactic antibiotics between 30 and 60 minutes before the operation, $14.9 \%$ at the time of incision, and $4.6 \%$ within 30 minutes following the operation (Table 3). There was no significant association between timing of surgical prophylaxis with SSI $\left(\chi^{2}=3.628, \mathrm{p}=\right.$ 0.258 ). Intraoperative re-dosing was given only in one case, where cefoperazone was used, when the duration of surgery exceeded six hours. There were 24 cases $(27.6 \%)$ where the surgery duration exceeded four hours and re-dosing was omitted, which was considered to be inappropriate. The rest of the surgery ended within four hours, which did not require redosing. The minimum cutoff point of four hours is considered to be based on our national guideline standard of more than half of the dosing interval; cefuroxime and augmentin should be re-dosed at four hours and cefoperazone at six hours.

A total of 67 patients $(77 \%)$ received antibiotics for $\leq 24$ hours; the remaining 20 (23\%) had antibiotics continued for $>24$ hours postoperatively (Table 3). Of the 20 patients who received antibiotics $>24$ hours where the antibiotics were continued as treatment, $12(60 \%)$ cases were for unknown reasons, whereas the remaining cases had documented SSI. The overall incidence of SSI was 12 out of the total 87 cases studied (13.8\%). It was noted that SSI mostly presented with redness $(83.3 \%)$ and tenderness $(75 \%)$ at the surgical site. Two cases had fever and positive culture of E. coli and Klebsiella pneumonia, respectively, and were extended spectrum betalactamases (ESBL) positive (Table 6).

\section{Discussion}

Our study showed that third-generation cephalosporin (cefoperazone) was the preferred antibiotic, and had an adherence rate of $78.2 \%$. In hepatobiliary, colorectal, and upper gastrointestinal (esophageal and gastroduodenal) surgery, most of the surgeons still preferred to use antibiotics with broader spectrum coverage, such as cefoperazone, despite second-generation cephalosporins (cefuroxime) or amoxicillin/clavulanate being one of the recommended choice of therapy. However, it is not known whether second-generation or $\beta$-lactam/ $\beta$-lactamase inhibitors could be substituted for third-generation cephalosporins as the preferred choice of antibiotics from the present cohort due to the limited cases using second-generation or $\beta$-lactam/ $\beta$-lactamase inhibitors. Most surgeons are comfortable with the use of thirdgeneration cephalosporins with broader antibacterial coverage, which are believed to lead to better reduction of SSI despite little evidence to support this. Metronidazole was commonly used in hepatobiliary and upper gastrointestinal surgery, which was inconsistent with the national guideline. In view of the higher incidences of SSI with the combination of cefoperazone and metronidazole compared to cefoperazone alone in hepatobiliary surgery, the addition of metronidazole might not be necessary. However, in upper gastrointestinal surgery, the use of cefoperazone and metronidazole led to fewer incidences of SSI as compared to cefoperazone alone, which somehow justified the use of metronidazole for anaerobes coverage in upper gastrointestinal surgery, even though this deviated from the guideline. Further research is needed to determine the appropriate use of metronidazole in hepatobiliary and upper gastrointestinal surgery in view of the limited number of cases in the present cohort.

Overuse of third-generation cephalosporins in surgical prophylaxis is alarming and has led to methicillin-resistant Staphylococcus aureus (MRSA) outbreaks [11]. The emergence of extended spectrum beta-lactamases (ESBL), vancomycin-resistant enterococci (VRE), and Clostridium difficile have also been widely reported as being related to the inappropriate use of third-generation cephalosporins [12]. Overuse of third-generation cephalosporins could be a potential threat in our setting and should lead to heightened vigilance in the selection of antibiotics for surgical prophylaxis. Surveillance on the culture and sensitivity for most commonly isolated pathogens should be carried out promptly to develop local antibiotic guidelines for the regulation of antibiotic usage in surgical prophylaxis in our setting.

Timing of the first dose of antibiotic therapy is important, as it aims to achieve adequate serum and tissue drug level for a period exceeding the duration of 
the operation to target the organisms likely to be encountered during the operation [13]. From the surveillance in our setting, nearly $20 \%$ of the surgical procedures were noted to have inappropriate timing of antibiotics prophylaxis either at the time of incision or postoperatively. A study by Stone et al. showed that the SSI rate was almost identical in surgical procedures where the first dose of antibiotic was given postoperatively and in those without antibiotic prophylaxis [14]. Thus, initiation of antibiotic prophylaxis postoperatively is not recommended [6]. Around $80 \%$ of the prophylactic antibiotics were administered within one hour prior to the surgical incision in our study, which were concordant with the guideline. Of this, around $75 \%$ of the antibiotics were given 30 minutes preoperatively. The adherence status was better as compared to a Dutch hospital (50\%), [7] and hospitals in the United States (55.7\%) [15].

Various studies have generated different findings about the optimal timing of surgical prophylaxis. A study by Classen et al. showed the reduction of SSI with the administration of antibiotics two hours before operation, [5] whereas other studies suggested the optimal timing of administration was within one hour and close to the time of incision (e.g. at anesthesia induction) $[2,7,16]$. Regarding the optimal window for surgical prophylaxis, studies have shown contradictory results as to whether the ideal time is fewer than 30 minutes or between 30 and 60 minutes prior to surgical incision. In the study by Weber et al., the SSI rate was found to be 1.95 times higher when antibiotic prophylaxis was administered fewer than 30 minutes compared with administration between 30 and 60 minutes prior to the surgery [17]. In contrast, the study conducted by Steinberg et al. showed an increased infection risk of 1.74 times with the increased time interval (fewer than 30 minutes versus between 30 and 60 minutes) between preoperative antibiotic and surgical incision [18]. As such, there is no consensus yet to advocate a more specific and optimal timing of prophylactic antibiotic administration. Our national guideline does not clearly specify the administration timeframe of surgical chemoprophylaxis aside from after the induction, which could create confusion and thus variability in clinical practices.

Intraoperative re-dosing of prophylactic antibiotics has been recommended for prolonged surgical procedures to ensure adequate coverage throughout the surgery, which reduces the risk of surgical site infections [19]. In the national antibiotic guideline, it is stated that re-dosing may be required when the duration of surgery is more than half of the usual dosing interval of the antibiotic. In the case of cefoperazone, for which the dosing interval is every twelve hours it should be re-dosed at six hours. Other antibiotics such as cefuroxime and augmentin should be re-dosed at four hours, as the usual dosing interval is every eight hours. However, there is discrepancy between the Malaysia National Antibiotic Guideline and the international guideline (the American Society of Health-System Pharmacists [ASHP] guideline) pertaining to the re-dosing parameter, which could have generated confusion. ASHP recommends readministering prophylactic antibiotics at one to two times the half-life of the antibiotic; for example, the re-dosing interval of cefuroxime should be at four hours, as the half-life is between one and two hours [20]. Cefoperazone, with a half-life of two hours, should be re-dosed at four hours, which is different from the six hours given as half of the usual dosing interval. Therefore, the recommendation of re-dosing based on half-life or dosing interval would lead to different timing in intraoperative antibiotic administration. In the case of cefoperazone, the difference of two hours could negatively impact the clinical outcome. A specific re-dosing interval should be specified in our guideline for better standardization and adherence. It would be more appropriate to recommend re-dosing according to the half-life of antibiotics, as it is more accurate to reflect the drop in drug concentration; this would thus ensure sufficient drug concentration throughout the surgery.

With regards to the duration of antibiotic prophylaxis, the national antibiotic guideline does not recommend that an additional dose be administered postoperatively. However, a prophylactic antibiotic administered within 24 hours following the end of the surgery is considered appropriate according to the surveillance parameter of infection control of the Malaysia Ministry of Health. In the present cohort, $23 \%$ of the cases had prophylactic antibiotics administered more than 24 hours postoperatively, with $60 \%$ for unknown reasons. This inappropriate practice was slightly higher compared to Dutch hospitals (18\%) [7]. In American hospitals, more than half of patients $(59.3 \%)$ had generally continued antibiotic prophylaxis for more than 24 hours after the surgery ended [15]. In fact, the optimal duration of antibiotic prophylaxis is not known, but is generally recommended to be discontinued within 24 hours following the operative procedure $[6,7,13]$ The 
unnecessary and prolonged use of prophylactic antibiotics is associated with the emergence of resistant pathogens and increased cost of medical care [21].

Several limitations of this study have been identified. The number of cases in the present study might not be sufficient to represent the overall adherence in surgical antibiotic prophylaxis of general surgical wards due to the short timeframe of the study. In addition, the surveillance was undertaken in the general surgical wards and excluded other surgery such as orthopedic, cardiothoracic, urology, obstetrics, and gynecology, making the data not generalizable to the various disciplines. Apart from this, the data retrieval was based on the written information in medical records, which might be confounded by personnel negligence in documentation and thus not truly reflect the real practice, especially regarding the timing of antibiotic prophylaxis.

\section{Conclusion}

The guideline adherence rates pertaining to the choice, timing, re-dosing, and duration of antibiotic usage in surgical prophylaxis were more than $70 \%$ for each respective parameter. Lack of awareness, ignorance, disagreement with the guideline, or different schools of thought could be the reasons for non-adherence; this requires further exploration. The development of an institutional antibiotic guideline could be more favorable and might gain better acceptance and agreement among surgeons as it would be suited to the local setting. A close collaboration among multidisciplinary teams, which involve surgical, anesthesia, microbiology, pharmacy, nursing, and the leadership team, can ensure effective guideline adoption and implementation in making this quality improvement program a success.

\section{Acknowledgements}

We would like to thank the Director General of Health Malaysia for permission to publish this paper and all doctors who contributed to the data collection of this study.

\section{References}

1. Committee on Antimicrobial Agents, Canadian Infectious Disease Society, Waddell TK, Rotstein OD (1994) Antimicrobial prophylaxis in surgery. Can Med Assoc J 151 Suppl 7: 925-931.

2. Geroulanos S, Marathias K, Kriaras J, Kadas B (2001) Cephalosporins in surgical prophylaxis. J Chemother 1 Suppl 1: 23-26.

3. McGowan JE (1991) Cost and benefit of perioperative antimicrobial prophylaxis: methods for economic analysis. Clin Inf Dis 13 Suppl 10: S879-S889.

4. Harbarth S, Samore MH, Lichtenberg D, Carmeli Y (2000) Prolonged antibiotic prophylaxis after cardiovascular surgery and its effect on surgical site infections and antimicrobial resistance. Circ 101: 2916-2921.

5. Classen DC, Evans RS, Pestotnik SL, Horn SD, Menlove RL, Burke JP (1992) The timing of prophylactic administration of antibiotics and the risk of surgical-wound infection. NEJM 326: 281-286.

6. Dellinger EP, Gross PA, Barrett TL, Krause PJ, Martone WJ, McGowan JE, Sweet RL, Wenzel RP (1998) Quality standard for antimicrobial prophylaxis in surgical procedures. Clin Inf Dis 18: 422-427.

7. Van Kasteren MEE, Kullberg BJ, Boer AS, Groot JM, Gyssens IC (2003) Adherence to local hospital guidelines for surgical antimicrobial prophylaxis: a multicentre audit in Dutch hospitals. J Antimicrob Chemother 51: 1389-1396.

8. National antibiotic guideline (2008) Ministry of Health Malaysia.

9. Mangram AJ, Horan TC, Pearson ML, Silver LC, Jarvis WR (1999) Guideline for prevention of surgical site infection. Infect Control Hosp Epidemiol 20: 250-278.

10. Policies and procedures on infection control (2010) Medical Development Devision, Quality Medical Care Section, Ministry of Health Malaysia.

11. Fukatsu K, Saito H, Matsuda T, Ikeda S, Furukawa S, Muto T (1997) Influences of type and duration of antimicrobial prophylaxis on an outbreak of methicillin-resistant staphylococcus aureus and on the Incidence of wound infection. Arch Surg 132 Suppl 12: 1320-1325.

12. Dancer SJ (2001) The problem with cephalosporins. J Antimicrob Chemother 48: 463-478.

13. Bratzler DW and Houck PM (2004) Antimicrobial prophylaxis for surgery: an advisory statement from the national surgical infection prevention project. Clin Inf Dis 38: 1706-1715.

14. Stone HH, Hooper CA, Kolb LD, Geheber CE, Dawkins EJ (1976) Antibiotic prophylaxis in gastric, biliary and colonic surgery. Ann Surg 184: 443-52.

15. Bratzler DW, Houck PM, Richards C, Steele L, Dellinger EP, Fry DE, Wright C, Ma A, Carr K, Red L (2005) Use of antimicrobial prophylaxis for major surgery. Arch Surg 140: 174-182.

16. DiPiro JT, Vallner J, Bowden TA, Clark BA, Sisley JF (1985) Intraoperative serum and tissue activity of cefazolin and cefoxitin. Arch Surg 120 Suppl 7: 829-832.

17. Weber WP, Marti WR, Zwahlen M, Misteli H, Rosenthal R, Reck S, Fueglistaler P, Bolli M, Trampuz A, Oertli D, Widmer AF (2008) The timing of surgical antimicrobial prophylaxis. Ann Surg 247: 918-926.

18. Steinberg JP, Braun BI, Hellinger WC, Kusek L, Bozikis MR, Bush AJ, Dellinger EP, Burke JP, Simmons B, Kritchevsky SB, Trial to Reduce Antimicrobial Prophylaxis Errors 
(TRAPE) Study Group (2009) Timing of antimicrobial prophylaxis and the risk of surgical site infection. Ann Surg 250: 10-16.

19. Bratzler DW, Dellinger EP, Olsen KM, Perl TM, Auwaerter PG, Bolon MK, Fish DN, Napolitano LM, Sawyer RG, Slain D, Steinberg JP, Weinstein RA (2013) Clinical practice guidelines for antimicrobial prophylaxis in surgery. Am J Health-Syst Pharm 70: 195-283.

20. Zanetti G, Giardina R, Platt R (2001) Intraoperative redosing of cefazolin and risk for surgical site infection in cardiac surgery. Emerg Infect Dis 7: 828-831.

21. Hecker MT, Aron DC, Patel NP, Lehmann MK, Donskey CJ (2003) Unnecessary use of antimicrobials in hospitalized patients. Arch Intern Med 163: 972-978.

\section{Corresponding author}

Ai Ling Oh

Department of Pharmacy, Sarawak General Hospital Jalan Tun Ahmad Zaidi Adruce, 93586 Kuching

Sarawak, Malaysia

Tel: 60-82-276612

Fax: 60-82-276703

E-mail: ohailing79@yahoo.com

Conflict of interests: No conflict of interests is declared. 